\title{
250nm Lithography with Step and Scan
}

\author{
HARRY SEWELL \\ SVG Lithography, \\ Wilton, CT 06897, USA.
}

This paper assesses the progress of Step and Scan systems towards "Production 250nm Lithography". Advantages of the Step and Scan technique are discussed.

The relationships between NA, wavelength, resolution, and depth of focus are examined. The lithographic performance of the new generation Deep-UV Step and Scan system with high-NA optics is presented. Data is provided from electrical probe linewidth metrology and SEM analysis. Linewidth control of $27 \mathrm{~nm}$ ( 3 sigma) is demonstrated over a large field size of $650 \mathrm{~mm}^{2}$ for $350 \mathrm{~nm}$ features. A $250 \mathrm{~nm}$ resolution capability is shown.

The family of Step and Scan systems, I-Line -- Deep-UV -- VeryDeep-UV, is reviewed in the context of progress towards $250 \mathrm{~nm}$ lithography. The impact of Phase Shift Masks is highlighted. Particular attention is paid to the effect of partial coherence on the depth of focus achievable with Phase Shift Masks. Examples of $250 \mathrm{~nm}$ lithography in a single level resist system are presented. It is indicated that optical lithography will continue to be the dominant lithographic technique down to sub-250nm lithography and that Step and Scan technique will be a critical factor in maintaining this dominance.

\section{Introduction}

The advanced semiconductor product is following the road map defined by Memory chip production (table 1 ).

\begin{tabular}{|c|c|c|c|}
\hline \hline Device & $\begin{array}{l}\text { Chip Size } \\
(\mathrm{mm} \times \mathrm{mm})\end{array}$ & Resolution & Total Overlay \\
\hline 16Mbit DRAM & $7 \times 14$ & $600 \mathrm{~nm}-450 \mathrm{~nm}$ & $120 \mathrm{~nm}$ \\
\hline 64 Mbit DRAM & $9.5 \times 19$ & $450 \mathrm{~nm}-350 \mathrm{~nm}$ & $100 \mathrm{~nm}$ \\
\hline 256 Mbit DRAM & $13 \times 26$ & $350 \mathrm{~nm}-250 \mathrm{~nm}$ & $80 \mathrm{~nm}$ \\
\hline 1Gbit DRAM & $18 \times 36$ & $250 \mathrm{~nm}-200 \mathrm{~nm}$ & $60 \mathrm{~nm}$ \\
\hline
\end{tabular}

Table 1. Estimated Lithographic Requirements of DRAM Production. 
At present, production technology is moving down to 400nm linewidths. Step and Scan technique has been developed for 350nm lithography and pre-production systems are now under testing for both performance and reliability. The early production units for 350nm lithography will begin shipping very shortly, including wafer track systems. The debate on the prospects for $250 \mathrm{~nm}$ lithography and 256MBit DRAMs has begun.

This paper will first discuss the advantages that the Step and Scan technology offers, and review the $350 \mathrm{~nm}$ lithographic performance that is currently available. The approaches that are being investigated to implement $250 \mathrm{~nm}$ Step and Scan lithography, including the impact of phase shift masks, are,then discussed.

\section{Advantages of Step and Scan}

Step and Scan systems are optical systems and therefore obey the same rules of optics concerning the relationship of wavelength and NA. Step and Scan systems are available in both Deep-UV and I-Line versions. These systems allow companies with device processing running with I-Line resists to transfer to the large exposure field step and scan system before switching over to Deep-UV. Hence becoming familiar with the Step and Scan technology.

Step and Scan has the advantage over conventional stepper in four significant ways. First, a Step and Scan system scans a long narrow slot image field to produce the exposed field, this scanning action has the effect of averaging the residual distribution in the lens as the image points pass through each lens field point in turn. This produces low distortion over a large field. Second, the multi-target alignment capability allows for high overlay accuracy and distortion fitting over a large field. Third, the terrain following focus control significantly reduces focus variations over a large field. Fourth, uniformity of illumination allows tight linewidth control over a large scanned field.

\subsection{Residual Field Distortion}

Step and Scan achieves low field distortion because it scan averages the optical distortion over a small field to produce even lower distortion. Figure 1 shows the distortion over the illuminated optical slit $22 \mathrm{~mm}$ by $5 \mathrm{~mm}$.

\author{
Average of 3 Fields \\ Field $5 \mathrm{~mm}$ by $21 \mathrm{~mm}$ \\ Statistics of Distortion \\ Max. Vec. $=66 \mathrm{~nm}$ \\ Ave. $V e c=19 \mathrm{~nm}$ \\ $X$ Std. Dev. $=16 \mathrm{~nm}$ \\ $Y$ Std. Dev. $=14 \mathrm{~nm}$
}

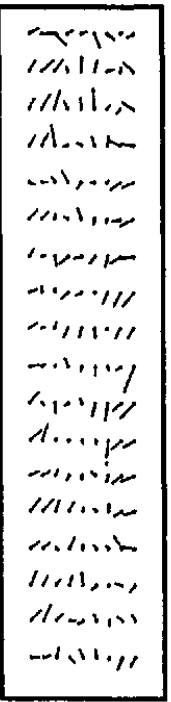

Figure 1. Static Field Distortion. 
Results for early optics indicate distortion of $66 \mathrm{~nm}$ ( $\max$. vectors), including the metrology errors. This is typical of a good "stepper lens". When this optical field is effectively scanned, the distortion is averaged for all points along the scan direction, this produces a dynamic optical distortion shown in figure 2 .

Static Distortion Averaged Along The Scan Direction (Computed)

Exposed 27 Feb 92, Condition 035 ALARM Data

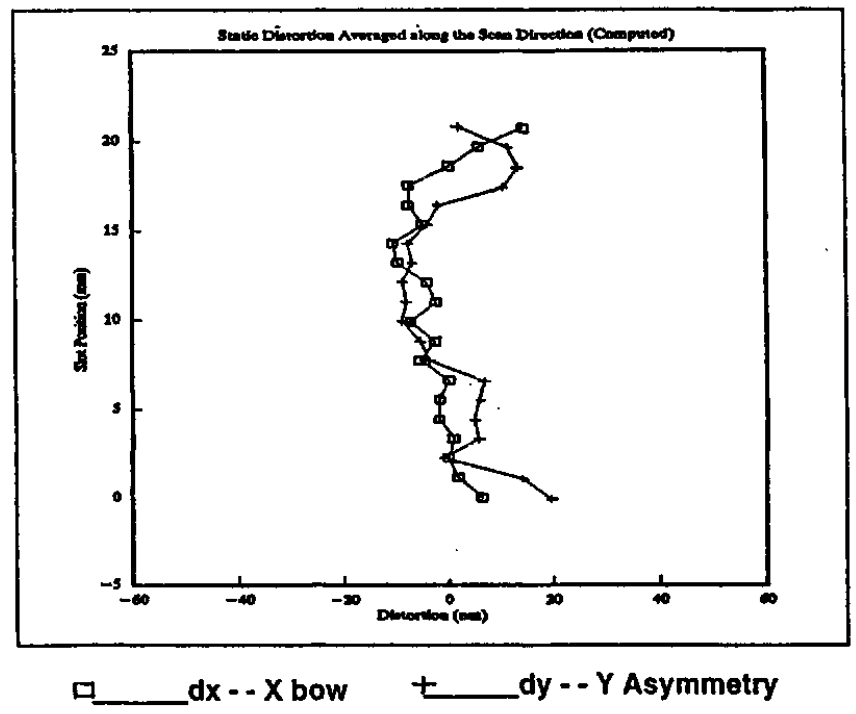

Figure 2. Dynamic Optical Distortion.

The effective optical distortion is reduced, in this case to less than 22nm, a threefold improvement. Of coarse, the dynamic field distortion in the case of Step and Scan is actually a product of the optical distortion and the distortion contributed by the scanning stages. Figure 3 shows the results obtained from the prototype system for the dynamic field distortion.

8 Field Average Field $29 \mathrm{~mm}$ by $20 \mathrm{~mm}$ Prototype System Reticle Errors Included

Statistics of Distortion

Max. Vec. $=41 \mathrm{~nm}$

Ave. $V e c=12 \mathrm{~nm}$

$X$ Std. Dev. $=6 \mathrm{~nm}$

Y Std. Dev. $=13 \mathrm{~nm}$

\begin{tabular}{|c|c|c|c|c|c|c|c|c|c|c|c|c|c|c|c|}
\hline & 1 & i. & & 1 & & 1 & & $!$ & 1 & i & 1 & & 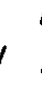 & 1 & \\
\hline & 1 & 1 & 1 & . & ' & 1 & 1 & . & • & • & & & & & \\
\hline 1 & $i$ & . & , & . & . & . & - & . & • & - & & & 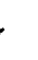 & & 1 \\
\hline 1 & ' & 1 & 1 & 1 & 1 & 1 & 1 & 1 & I & $!$ & & & & • & $\checkmark$ \\
\hline ' & , & 1 & 1 & 1 & 1 & 1 & 1 & 1 & 1 & 1 & & & 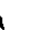 & & ' \\
\hline , & 1 & 1 & 1 & 1 & 1 & 1 & 1 & 1 & 1 & 1 & & & & & ' \\
\hline ' & • & 1 & 1 & 1 & $i$ & $\cdot$ & 1 & , & ' & 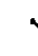 & & & 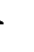 & & · \\
\hline 1 & - & 1 & 1 & , & ' & • & , & , & 1 & 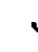 & & 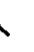 & & & . \\
\hline$r$ & . & . & , & a & . & . & . & $\checkmark$ & • & . & & & & & 1 \\
\hline 1 & 1 & . & . & . & . & . & - & . & . & . & & & t & & $\checkmark$ \\
\hline 1 & . & 1 & . & a & . & . & . & . & . & . & & & & & 1 \\
\hline 1 & 1 & . & . & . & . & v & > & - & $\checkmark$ & 1 & & & & & 1 \\
\hline
\end{tabular}

Figure 3. Dynamic Field Distortion. 
Even on the prototype, 40nm was achieved with the $\mathrm{X}$-axis distortion being less than $20 \mathrm{~nm}$. Indications are that this can be further reduced to less than $30 \mathrm{~nm}$ with the correction of the slight bow distortion. These distortion results demonstrate the unique property of Step and Scan to produce large exposure fields with low levels of distortion. This performance will support a $60 \mathrm{~nm}$ total overlay budget on a $250 \mathrm{~nm}$ tool.

\subsection{Overlay}

Figure 4 shows the typical exposure layout used for overlay testing. 36 fields are used each $22 \mathrm{~mm}$ by $32.5 \mathrm{~mm}$.

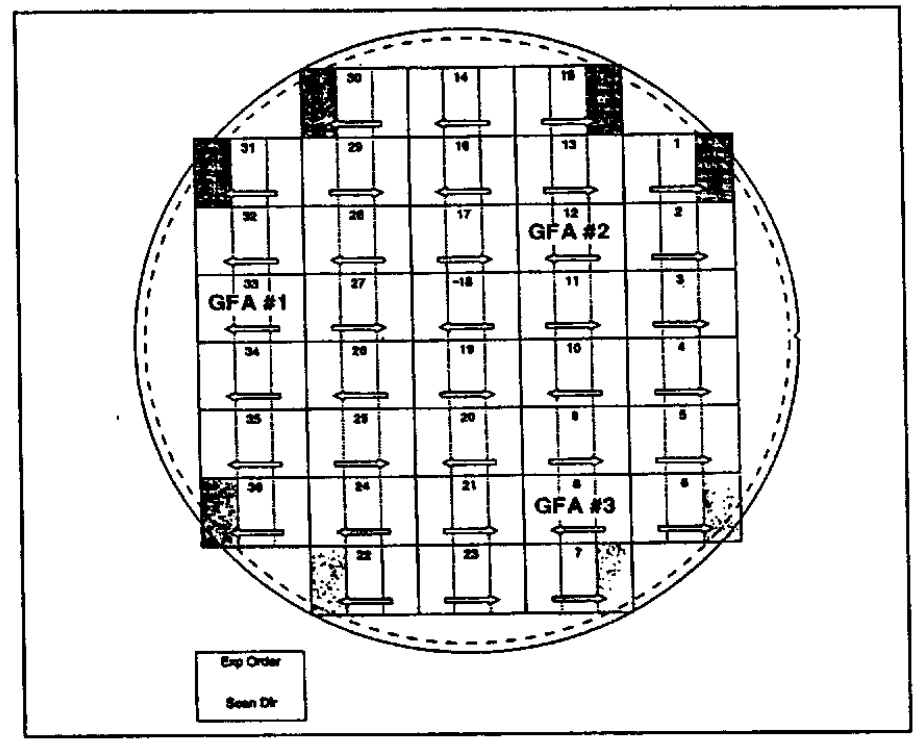

Figure 4. 36 Field 6-Term Global Layout for 8" Wafers.

These fields are produced by scanning the wafer and reticle through an illuminated optical field $5 \mathrm{~mm}$ by $22 \mathrm{~mm}$. Scan directions are shown. For Global Alignment three or more fields are pre-scanned and six alignment terms fitted: X-Offset; $Y$ Offset; Skew; Rotation; X-Mag; Y-Mag. From the position of these die the grid is calculated. Results for total overlay accuracy on etched oxide wafers from the prototype testing are shown in figure 5.

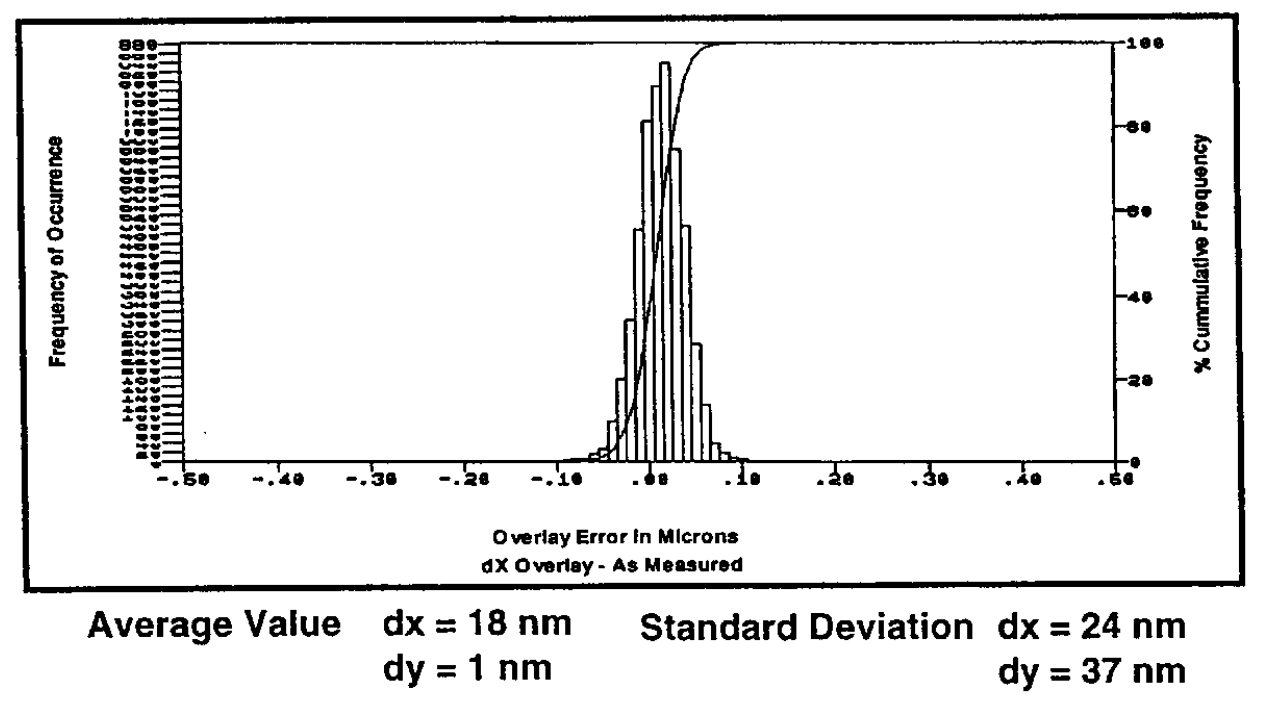

Figure 5. Step and Scan Overlay (Prototype System). 
Approximately 100nm (mean plus 3 sigma) is indicated. Further testing on preproduction units, in which wafer and reticle stage laser mirror mapping has been introduced indicates that approximately $85 \mathrm{~nm}$ (mean plus 3 sigma) is achieved. $60 \mathrm{~nm}$ (mean plus 3 sigma) is expected to be achieved on production units which have improved stage control algorithms. The success in meeting this $60 \mathrm{~nm}$ goal will be a major success in the realization of a $250 \mathrm{~nm}$ Step and Scan system.

\subsection{Dynamic Focus}

The Step and Scan system focuses on the fly. Typical focus contours over a field are shown in figure 6.

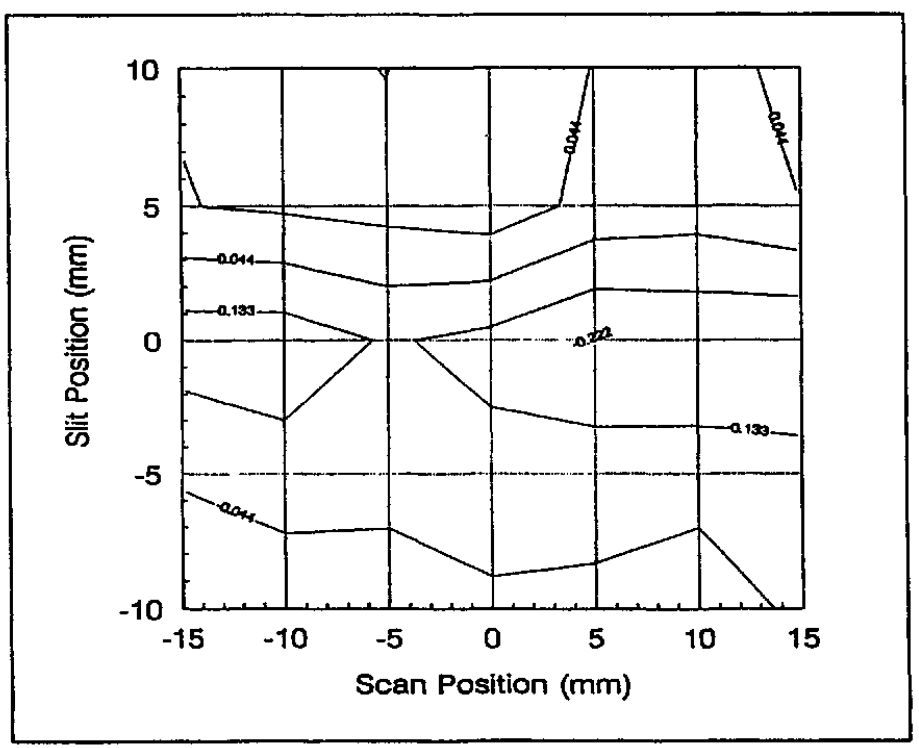

Figure 6. Dynamic Focus Uniformity on a Silicon Wafer.

Focus is very tightly controlled in the scan direction, better than $100 \mathrm{~nm}$. The predominant focus variation is in the cross-scan direction, in this case approximately $200 \mathrm{~nm}$ and represents some field curvature in the prototype optics. This cross-scan field curvature is being progressively reduced and is expected to eventually be less than $100 \mathrm{~nm}$ in production units. The total focus budget for the current preproduction units is less than $\pm 0.4 u m$, including edge fields. Improvements in optical field curvature to $100 \mathrm{~nm}$ will allow the total focus budget to be reduced to $<200 \mathrm{~nm}$. This will be important advantage for the realization of a $250 \mathrm{~nm}$ exposure tool using Step and Scan.

\subsection{Linewidth Control}

The current step and Scan tools are $0.50 \mathrm{NA}$ and both Deep-UV and I-line versions are available. The specified resolution for the I-line tool is $500 \mathrm{~nm}$ and the Deep-UV tool is 350nm. We are using electrical probe metrology to assess both resolution and linewidth control performance. We measure both grouped and isolated lines. Results shown in figure 7 from the prototype system indicate a depth of focus of over one micron for grouped lines with good linewidth control. 
$350 \mathrm{~nm}$ Lines Using 2208 Positive Resist Resist.

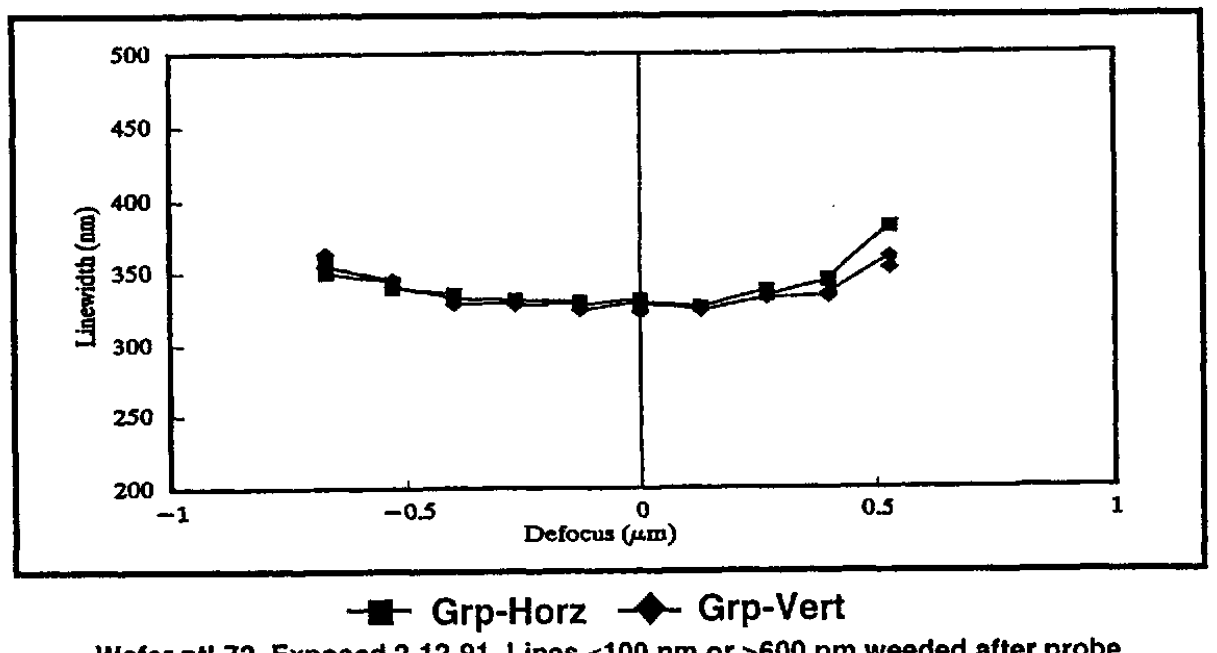

Wafer pti-72. Exposed 2-12-91. Lines $<100 \mathrm{~nm}$ or $>600 \mathrm{~nm}$ weeded after probe.

Figure 7. Linewidth through Focus for Grouped Lines

Analysis of the linewidth control through a focus range of \pm 0.4 um indicates remarkable results for the prototype system. Histograms of linewidth control shown in Figure 8 and 9 indicate that better than 35nm ( 3 sigma) values were obtained in etched polysilicon for isolated or grouped lines, this includes metrology and reticle errors.

Over \pm 0.4 um Focus Range, 2208 Positive Resist

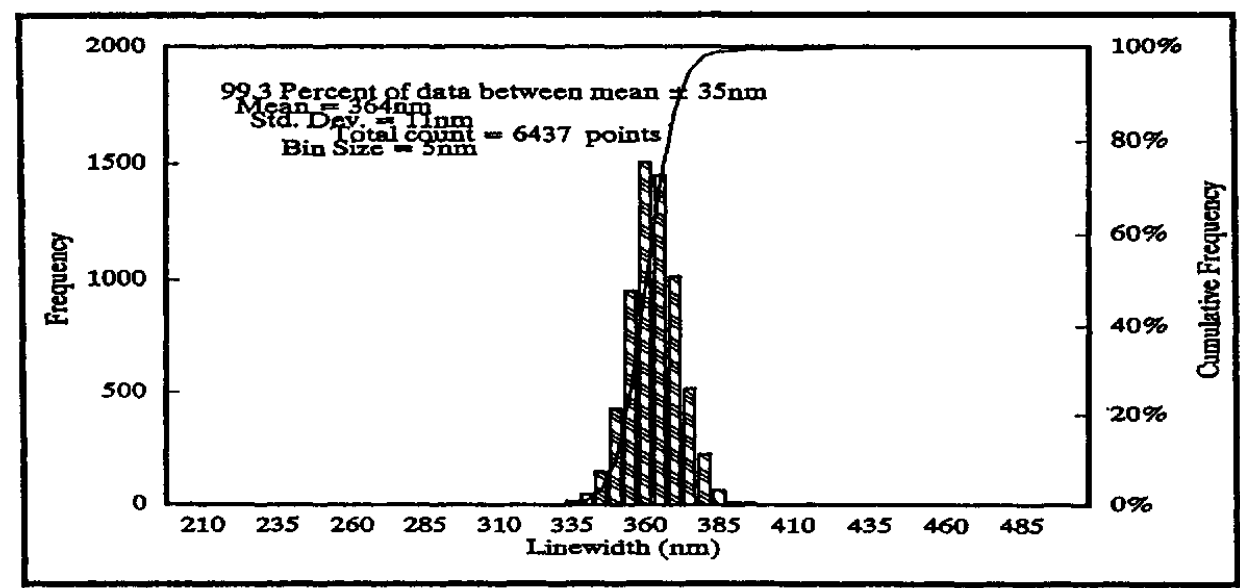

Wafer ptl-72. Exposed 2-12-91. Lines $<100 \mathrm{~nm}$ or $>600 \mathrm{~nm}$ weeded after probe.

Figure 8. 350nm Linewidth Control Histograms -- Isolated Lines. 
Over \pm 0.4 um Focus Range, 2208 Positive Resist

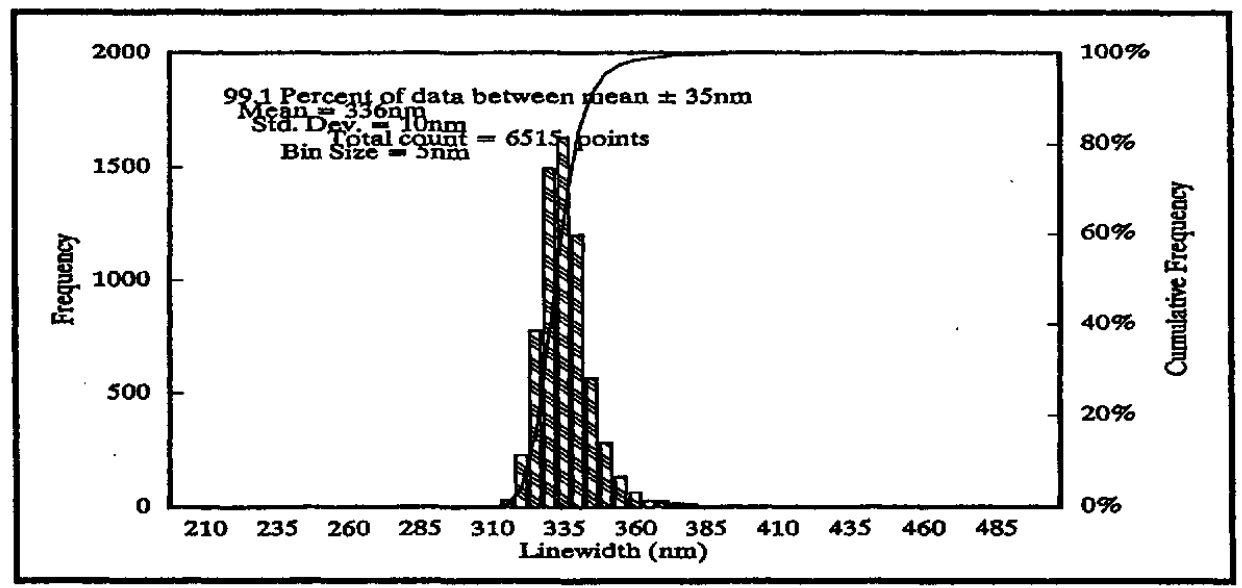

Wafer ptl-72. Exposed 2-12-91. Lines $<100 \mathrm{~nm}$ or $>600 \mathrm{~nm}$ weeded after probe.

Figure 9 350nm Linewidth Control Histograms -- Grouped Lines.

The high degree of linewidth control demonstrated by Step and Scan is in part due to the tight control of focus and illumination that a Step and Scan system can achieve. The illumination along the scanning slit is controlled to better than $\pm 1 \%$. We did note an offset between the groped and isolated average linewidths of $28 \mathrm{~nm}$. We have investigated this phenomena and concluded that it is predicted by the theory of optics. It is an important effect that we are studying for $250 \mathrm{~nm}$ lithography.

\section{Progress towards production 250nm Lithography}

Two optical approaches are available for a $250 \mathrm{~nm}$ resolution tool: High NA or Very-Deep-UV (193nm). Both approaches are being pursued. Depth of focus is the issue. Figure 10 indicates the estimated depth of focus for current and future systems.

\section{Grouped Lines Just Opened in Positive Resist}

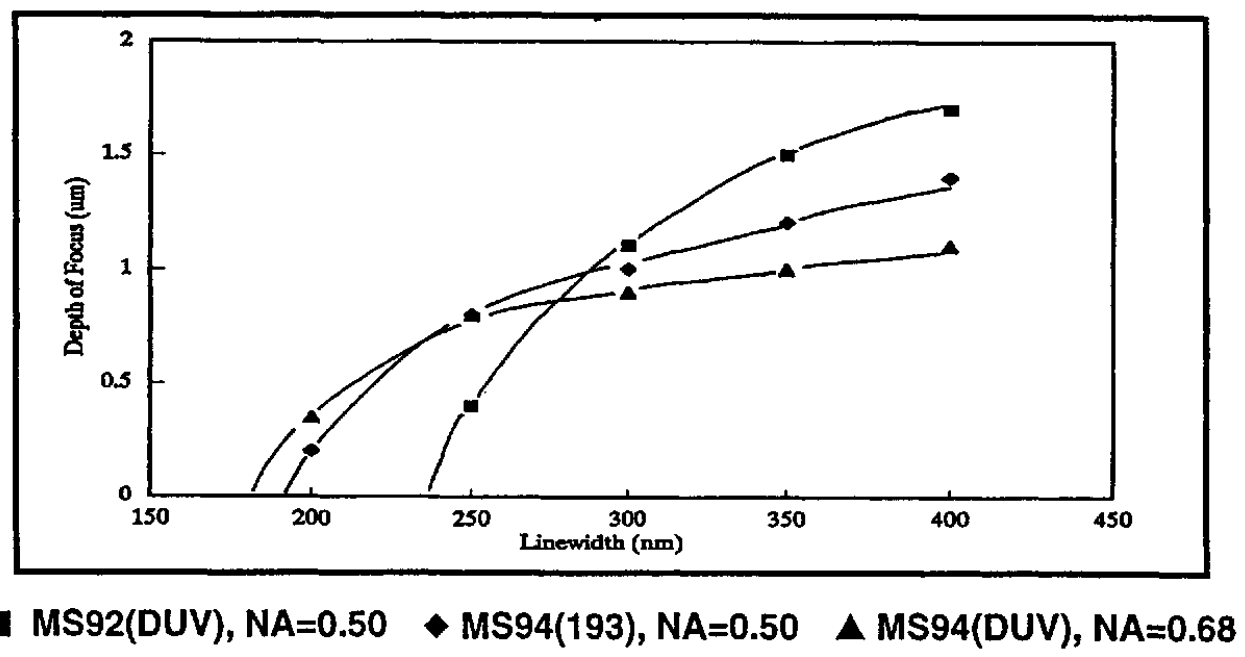

Figure 10. Resolution and Depth of Focus. 
It is expected that the nominal depth of focus for either a 193nm system or a VeryHigh-NA system at $250 \mathrm{~nm}$ will be approximately $0.8 \mathrm{um}$, but this is subject to processing. Technologies such as surface imaging, bi-level resist, annular illumination, and phase-shift masks will increase this depth of focus. Phase shift techniques will be the prime technique as annular illumination produces some degredation in the quality of the aerial image which may be critical at 250nm resolution. Combination of phase shifting and annular illumination may be more effective. Using the etched phase shifter technology and the Leverson alternating phase shifter technique the depth of focus can be significantly enhanced when highly coherent illumination is used. The depth of focus at $250 \mathrm{~nm}$ resolution using a $0.50 \mathrm{NA}$ Deep-UV Step and Scan system is enhanced from 0.4 um two to threefold depending upon the partial coherence used. Figure 11 shows that for a partial coherence $<0.3$, over 1.25um depth of focus is expected (note, for partial coherence $>0.6$ there is little improvement in depth of focus because the phase shift effect is derived from the interference of out of phase adjacent coherent images).

\section{LAMBDA $=248 \mathrm{~nm}, \mathrm{NA}=0.50$, Grouped Lines}

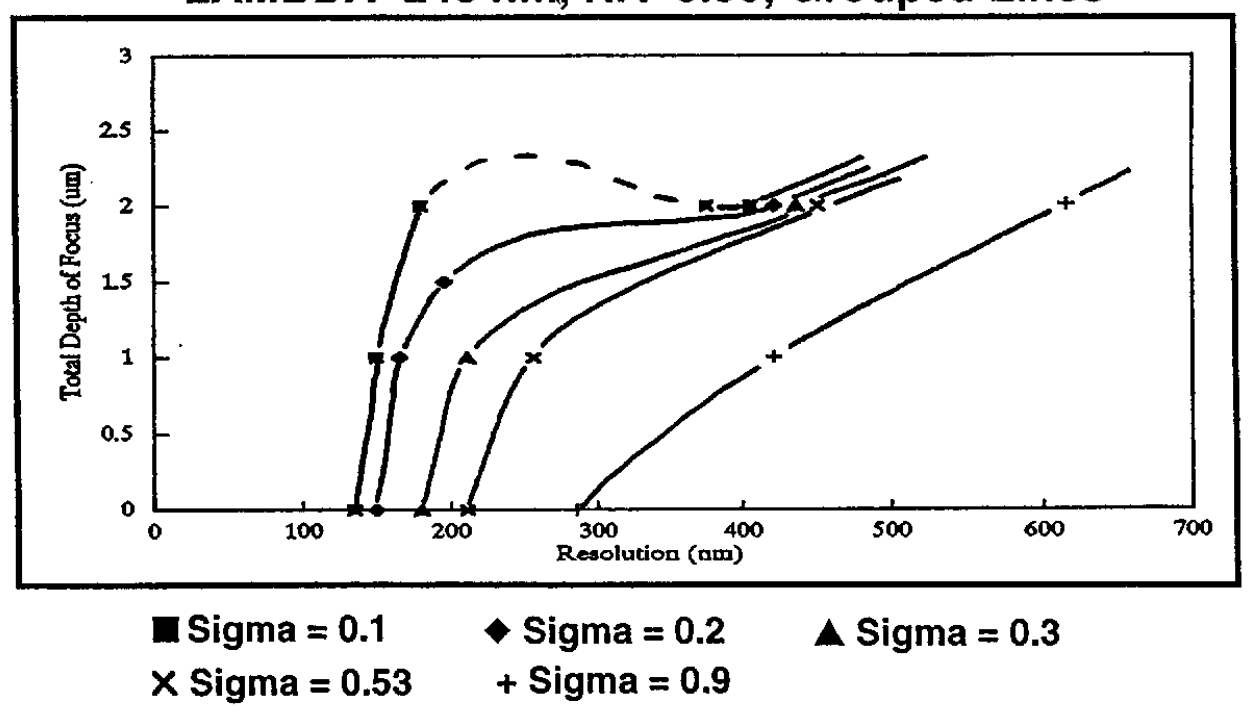

Figure 11. Depth of Focus for Grouped Lines using a Phase-shift Mask (NA=0.50)

The effect of phase shift masks on resolution can be clearly observed if we plot the the clear line intensity and the opaque line intensity for grouped line patterns of various linewidths. Figure 12 is plotted for a standard mask used on a 0.50NA Step and Scan systems at I-line, DUV, and VDUV. It is clear to see that the $60 \%$ process point indicates the resolution capabilities of the I-line, DUV, and VDUV systems to be $400 \mathrm{~nm}, 280 \mathrm{~nm}$ and $230 \mathrm{~nm}$ respectively. This shows that an I-line system will not be able to achieve $250 \mathrm{~nm}$ resolution in production, unless the NA is substantially higher than 0.50 or phase shift masks are used.

If phase shift masks are used then a 0.50 NA Step and Scan I-line system will be able to achieve $250 \mathrm{~nm}$ resolution (Figure 13). 200nm will be achieved on the DUV Step and Scan system and $160 \mathrm{~nm}$ on the Very-Deep-UV system. Early testing of phase Shift masks on the DUV 0.50 NA prototype system indicate that $250 \mathrm{~nm}$ is readily achieved as shown in figure 14. The available depth of focus was over $0.8 \mathrm{um}$ using a partial coherence of 0.3 . 


\section{$\mathrm{NA}=0.50, \mathrm{SIGMA}=0.5, \mathrm{FOCUS}=0.0 \mathrm{um}$, Standard Mask}

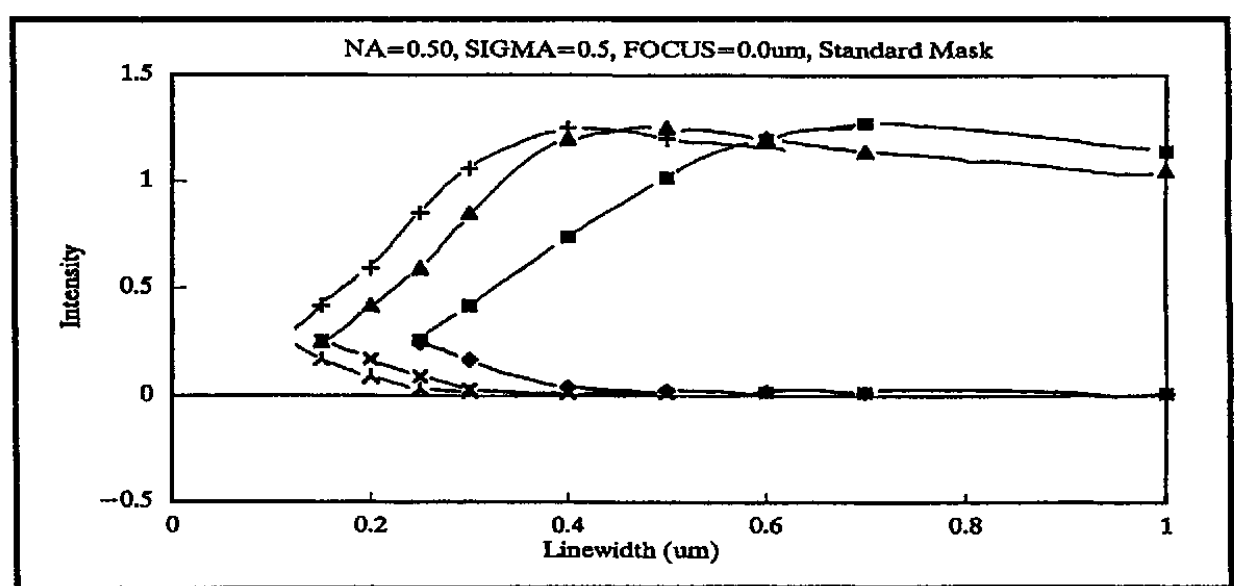

Clear Line Ints. (I-Line) $\diamond$ Opaque Line Ints. (I-Line) $\Delta$ Clear Line Ints. (DUV) $X$ Opaque Line Ints. (DUV) + Clear Line Ints. (VDUV) ᄉ Opaque Line Ints. (VDUV)

Figure 12. Image Contrast (I-line, DUV, VDUV) using a Standard Mask.

$\mathrm{NA}=0.50, \mathrm{SIGMA}=0.3$, FOCUS $=0.0$ um, Phase Mask

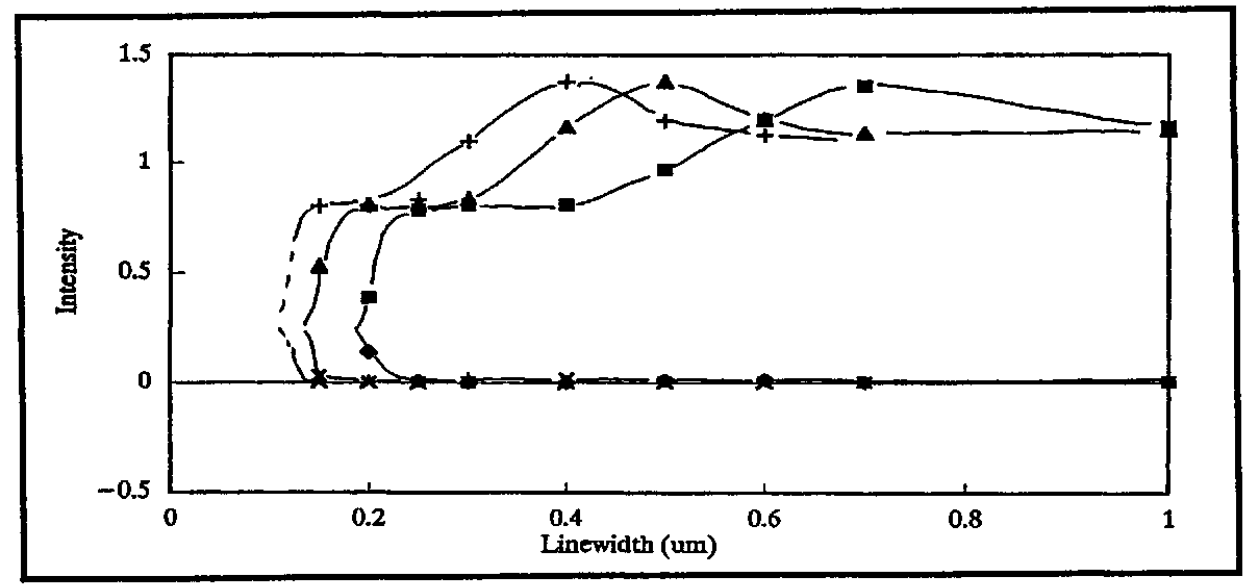

Clear Line Ints. (I-Line) $\diamond$ Opaque Line Ints. (I-Line) $\Delta$ Clear Line Ints. (DUV) $X$ Opaque Line Ints. (DUV) + Clear Line Ints. (VDUV) ᄉ Opaque Line Ints. (VDUV)

Figure 13. Image Contrast (I-line, DUV, VDUV) using a Phase-shift Mask. 


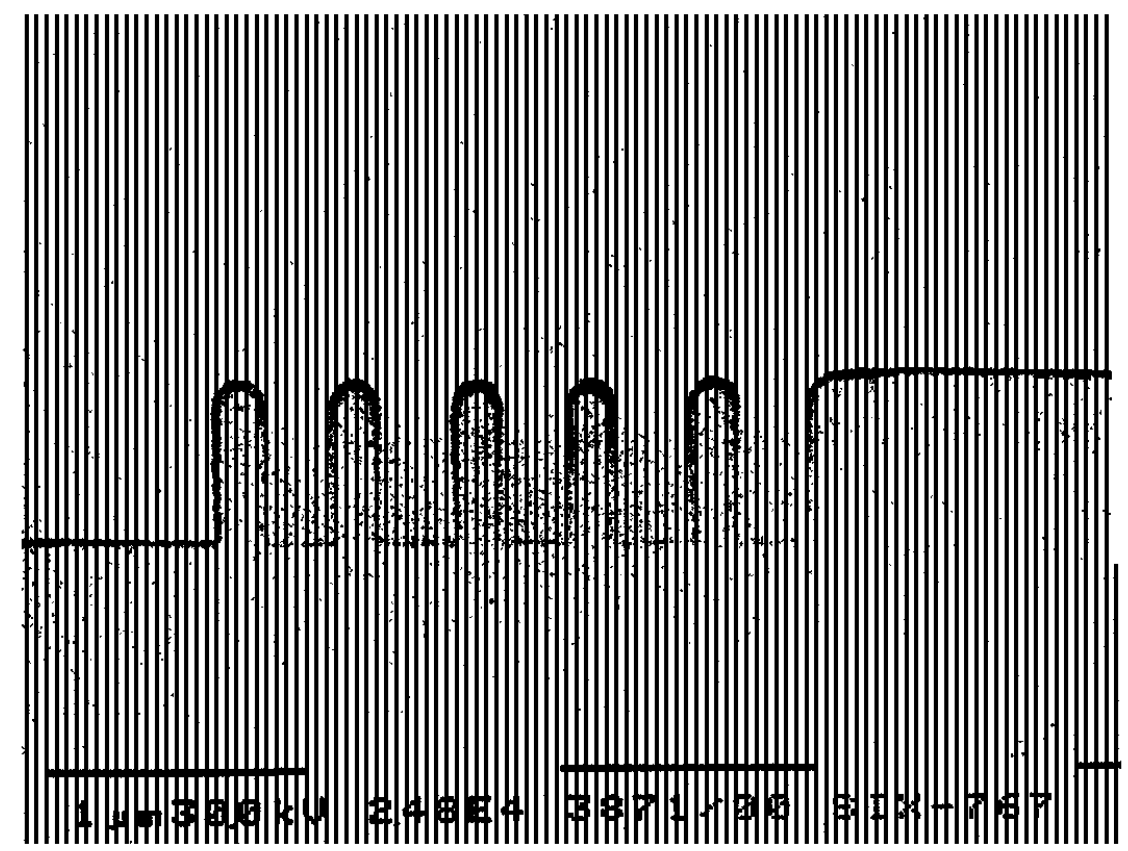

Figure 14. SEM Photograph of 250nm Grouped Lines Exposed on DUV Step and Scan using a Phase-shift Mask. (NA=0.50, Sigma=0.3)

4. Conclusions.

250nm resolution will be achieved with Step and Scan using Very-High-NA optics or $193 \mathrm{~nm}$ illumination on a Step and Scan system. The first of the 193nm systems will begin testing within six months. The availability of production quality phase shift masks will enhance the depth of focus of these systems for periodic patterns and allow current 0.50 NA I-line and DUV Step and Scan systems to achieve $250 \mathrm{~nm}$ resolution. 